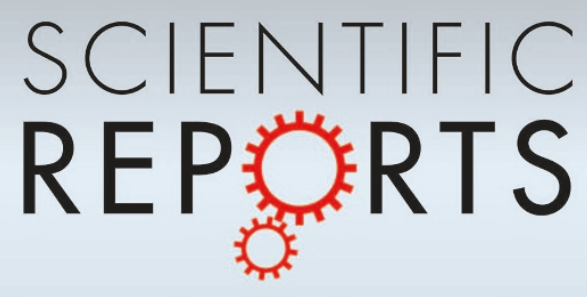

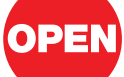

SUBJECT AREAS:

NONUINEAR OPTICS

OPTICAL MANIPULATION AND TWEEZERS

APPLIED PHYSICS

ULTRAFAST LASERS

Received

27 September 2012

Accepted

20 November 2012

Published

20 December 2012

Correspondence and requests for materials should be addressed to H.T.W. (htwang@nju. edu.cn; htwang@ nankai.edu.cn)

\section{Taming the Collapse of Optical Fields}

\author{
Si-Min Li', Yongnan Li' ', Xi-Lin Wang' ' Ling-Jun Kong' ', Kai Lou', Chenghou Tu' , Yongjun Tian³ \\ \& Hui-Tian Wang ${ }^{1,2}$
}

${ }^{1}$ MOE Key Laboratory of Weak Light Nonlinear Photonics and School of Physics, Nankai University, Tianjin 300071 , China,
${ }^{2}$ National Laboratory of Solid State Microstructures, Nanjing University, Nanjing 210093 , China, ${ }^{3}$ State Key Laboratory of
Metastable Materials Science and Technology, Yanshan University, Qinhuangdao 066004, China.

Field collapse, which occurs in various nonlinear systems, has attracted much attention, owing to its universality, complexity, and applicability. A great challenge and expectation is to achieve the controllable and designable collapsing pattern. Here we predict theoretically and demonstrate experimentally the novel collapsing behaviors of the vector optical fields in a self-focusing Kerr medium. Surprisingly, the results reveal that the collapse of the vector optical field is controllable and designable by engineering the distribution of hybrid states of polarization, and has the robust feature insensitive to the random noise. Our idea has its significance which it opens a new window for manipulating the optical field and the different kinds of field, and then facilitates to push the related researches.

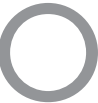

ptical field will undergo collapse at the input power above a certain critical power when an intense laser field propagates in a transparent self-focusing medium ${ }^{1-5}$. The high intensity as the collapse exacerbates will induce other nonlinear effects, such as multiphoton and saturable absorptions, to counterbalance self-focusing, resulting ultimately in filamentation ${ }^{3-12}$. Filamentation is an interesting and important topic due to its underlying physics and practical implication in many branches of physics ${ }^{13-18}$. A great challenge and expectation is to tame the randomicity and then to realize the controllable and even designable filamentation. For this purpose, several methods have been proposed such as, controlling the input power and divergence angle ${ }^{19}$, shaping the field profile ${ }^{20,21}$, using amplitude/phase mask ${ }^{22-25}$, and introducing spatial regularization ${ }^{26}$.

Polarization, as an intrinsic and fundamental vectorial nature of light, plays an indispensable role in the interaction of light with matter. Trisorio and $\mathrm{Hauri}^{27}$ demonstrated the spatial control on two filaments using circularly polarized femtosecond laser pulses. Fibich and Ilan ${ }^{3,28}$ presented a theoretical prediction that under the nonparaxial condition solely, the vectorial (polarization) effect of the homogeneously linearly-polarized field can lead to two filaments. However, polarization and its diversity of spatial distribution are not yet utilized fully. For the field collapse and the multiple filamentation, besides the self-focusing effect which is a requirement for the propagation medium, a prerequisite is that the optical field should be axial-symmetry breaking in and of itself. Therefore, to achieve the active control for the field collapse and the multiple filamentation, the key points are that the axial-symmetry breaking must be controllable and even designable, and the randomicity must be effectively suppressed simultaneously. A question is raised that whether the state of polarization (SoP) of light, as a degree of freedom, can be used to break the axial symmetry of optical field and to then availably control the field collapse and multiple filamentation. Vector optical fields with spatially inhomogeneous SoP distribution ${ }^{29,30}$ provide indeed such an opportunity and possibility, due to the designability and diversity of SoP distribution.

\section{Results}

In this paper, we present an idea that the axial-symmetry breaking of optical field by engineering the azimuthallyvariant SoP distribution controls the collapsing filamentation. We perform, to the best of our knowledge, the first study of the collapse of the vector optical fields in a self-focusing Kerr medium. The experimental results are in excellent agreement with our theoretical prediction. Some novel features are found, including the controllable, designable and robust features. The present study can contribute further to the study of collapsing filamentation in other nonlinear systems and other kinds of fields.

An important route breaking the axial symmetry of optical field is based on the azimuthally-variant hybridpolarized vector field (AV-HP-VF), which is a family of vector optical field with a "top-hat-like" intensity profile excluding a central singularity caused by the polarization uncertainty ${ }^{30}$. Under the weak focusing condition, the focused AV-HP-VF exhibits a uniform-intensity focal ring instead of a focal $\operatorname{spot}^{30}$. Its radially variant amplitude $A(r)$ can be described by the generalized hypergeometric function; nevertheless, as a well approximation, $A(r)$ can take the form of $A(r)=a_{0} r \exp \left(-r^{2} / 2 r_{0}^{2}\right)$, where $r_{0}$ is the radius of the focal ring. Inasmuch as the weak focusing 
does not change the SoP distribution, the focused AV-HP-VF in a polar coordinate system $(r, \phi)$ can be written as

$$
\mathbf{E}(r, \phi)=A(r)\left[\cos \delta|H\rangle+e^{-j \pi / 2} \sin \delta|V\rangle\right],
$$

where $\delta=m \phi+\phi_{0}$ ( $m$ is the topological charge and $\phi_{0}$ is the initial phase), and $|H\rangle$ and $|V\rangle$ are two unit vectors indicating the horizontally and vertically polarized components, respectively. For the AVHP-VF with $m$ and $\phi_{0}$, the local linear polarizations are located at a series of azimuthal positions $\phi^{l i n}(n)=n \pi / 2 m-\phi_{0} / m$, whereas local circular polarizations are at a series of $\phi^{c i r}(n)=(2 n+1) \pi / 4 m-\phi_{0} / m$ (where $n=0,1, \ldots, 4 m-1$ ). Differently from the optical vortex ${ }^{31}$, the AV-HP-VFs carry no orbital angular momentum though they also have a strictly zero field at the central singularity ${ }^{32}$. By contrast, an azimuthally-variant linearly-polarized vector field (AV-LP-VF) can be written as follows

$$
\mathbf{E}(r, \phi)=A(r)[\cos \delta|H\rangle+\sin \delta|V\rangle] .
$$

Compared Eq. (2) with Eq. (1), it can be found the unique difference that the horizontally and vertically polarized components have a phase difference of $-\pi / 2$ for AV-HP-VF, while are in phase for AV-LP-VF.

To theoretically explore the nonlinear propagation behavior of a vector optical field in a Kerr medium, the $(2+1)$-dimensional vectorversion nonlinear Schrödinger (NLS) equation should be used. Under the slowly varying amplitude approximation, the vector-version NLS equation can be divided into a pair of coupled NLS equations for two orthogonal horizontal and vertical components

$$
\begin{aligned}
& \frac{\partial \psi_{H}}{\partial \zeta}=\frac{j}{4} \nabla_{\perp}^{2} \psi_{H}+\frac{j \alpha P}{3 P_{C}}\left(3\left|\psi_{H}\right|^{2} \psi_{H}+2\left|\psi_{V}\right|^{2} \psi_{H}+\psi_{V}^{2} \psi_{H}^{*}\right), \\
& \frac{\partial \psi_{V}}{\partial \zeta}=\frac{j}{4} \nabla_{\perp}^{2} \psi_{V}+\frac{j \alpha P}{3 P_{C}}\left(3\left|\psi_{V}\right|^{2} \psi_{V}+2\left|\psi_{H}\right|^{2} \psi_{V}+\psi_{H}^{2} \psi_{V}^{*}\right),
\end{aligned}
$$

where $\psi_{q}(q=H, V)$ is the nondimensional $q$-component normalized by the total field, $\psi_{q}(\rho, \phi ; \zeta)=E_{q}(\rho, \phi ; \zeta) /$ $\sqrt{\iint\left[\left|E_{H}(\rho, \phi ; \zeta)\right|^{2}+\left|E_{V}(\rho, \phi ; \zeta)\right|^{2}\right] \rho d \rho d \phi} . \quad \rho=r / r_{0}, \quad \zeta=z / L_{d}$ $\left(L_{d}=\pi r_{0}{ }^{2} / \lambda\right)$ are the nondimensional cylindrical coordinates. $P=2 n_{0} \varepsilon_{0} c \iint\left[\left|E_{H}(r, \phi ; z)\right|^{2}+\left|E_{V}(\rho, \phi ; z)\right|^{2}\right] r d r d \phi$ is the power, $P_{C}=$ $\alpha \lambda^{2} / 4 \pi n_{0} n_{2}$ is the critical power for self-focusing, $n_{0}$ and $n_{2}$ are the linear and nonlinear refractive indices of the Kerr medium, $\alpha$ is a constant dependent on the initial field shape ${ }^{33}, c$ is the speed of light in vacuum, and $\lambda$ is the wavelength, respectively. On the righthand side of Eq. (3), the first term represents the contribution from the diffraction described by the transverse Laplacian $\nabla_{\perp}^{2}=\partial^{2} / \partial \rho^{2}+\frac{1}{\rho} \partial / \partial \rho+\frac{1}{\rho^{2}} \partial^{2} / \partial \phi^{2}$, and the second term is from the Kerr nonlinearity.

We performed a large number of simulations using Eq. (3) under $P$ $=10 P_{C}$ in a self-focusing Kerr medium. When the spatial random noise is added in simulation, the random noise with a level of $\pm 10 \%$ amplitude is used. The first and second rows in Fig. 1 illustrate the simulation results for the collapsing behavior of the focused AV-HPVF (with $m=1$ and $\phi_{0}=0$ ) described by Eq. (1), without and with random noise, respectively. By comparison, the third and fourth rows in Fig. 1 show the simulation results for the AV-LP-VF (with $m=1$ and $\phi_{0}=0$ ) described by Eq. (2), which is the well-known radially-polarized vector field $(\mathrm{RP}-\mathrm{VF})^{29}$, without and with random noise. Although both AV-HP-VF and RP-VF display the same focusing ring, their nonlinear propagation behaviors are quite different. Figure 2 shows the simulated collapsing patterns of the AV-HP-VFs with $m=1\left(\phi_{0}=0\right.$ and $\left.\pi / 4\right)$ and $m=2\left(\phi_{0}=0\right.$ and $\left.\pi / 8\right)$ when the spatial random noise is added. Summarizing all the simulation results performed, we can draw following conclusions. For the AVHP-VF with the given $m$ and $\phi_{0}$, (i) the azimuthally-variant hybrid
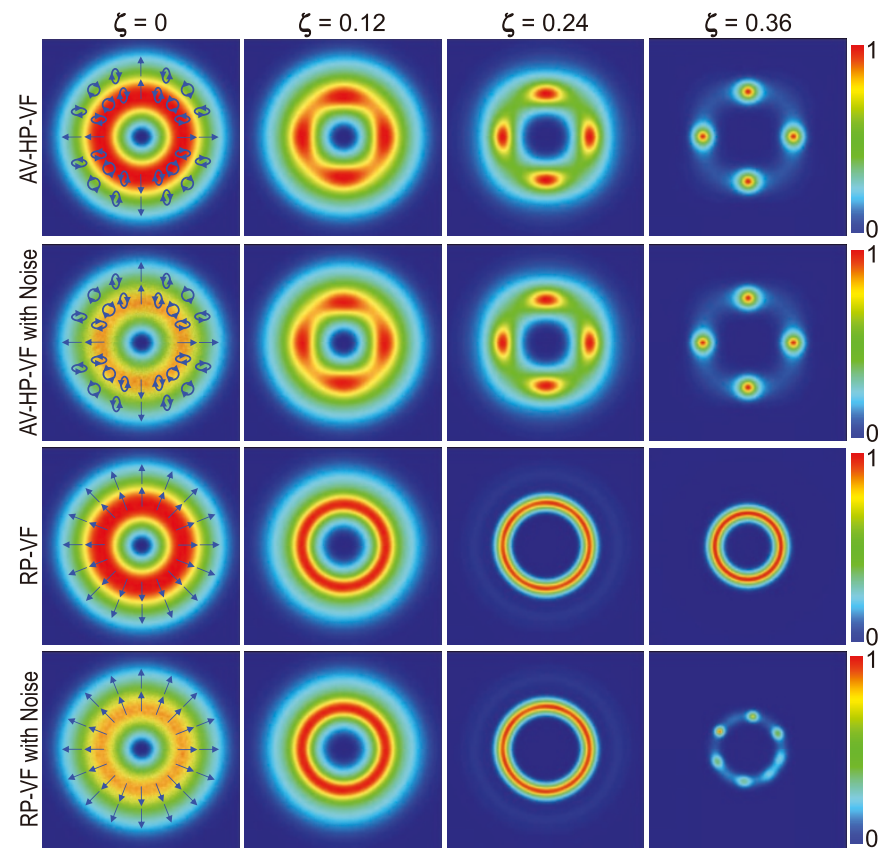

Figure 1 | Simulated nonlinear propagation behaviors of AV-HP-VF with $m=1$ and $\phi_{0}=0$ in equation (2). The first and second rows correspond to the cases without and with random noise, respectively. For comparison, the RP-VFs without and with random noise are also shown in the third and fourth rows. The four columns (from left to right) correspond to four propagation distances $(\zeta=0,0.12,0.24,0.36)$. The SoP distributions are also shown in the first column.

SoP distribution leads to the axial-symmetry breaking; (ii) the field undergoes the collapse to converge into the deterministic filaments; (iii) the number of filaments is $4 m$ depending solely on $m$; (iv) the filaments are always located at the azimuthal positions $\phi^{\text {lin }}(n)$, where their local SoPs are linearly polarized; (v) the collapsing pattern exhibits a $4 m$-fold rotation $\left(C_{4 m}\right)$ symmetry; and (vi) the collapsing patterns persist among simulations, with and without different random noise, implying that collapsing patterns are insensitive to noise. Meanwhile, for the AV-LP-VFs, (i) the purely ideal AV-LP-VF always remains in the axially symmetric ring, indicating that the purely ideal AV-LP-VF cannot converge to the deterministic multiple filaments; and (ii) the collapsing filaments produced by AV-LPVF with the random noise has the uncertainty, similar with the collapses of optical vortices $^{31}$ and polarization vortices ${ }^{34}$.

To experimentally confirm our theoretical prediction and numerical simulations, all the required vector fields with different $m$ and $\phi_{0}$ were created by using the experimental scheme ${ }^{30}$. The created AVHP-VFs and AV-LP-VFs were weakly focused into a uniformintensity focal ring and then incident into the $\mathrm{CS}_{2}$ cell with a length of $10 \mathrm{~mm}$, by an achromatic lens with a focal length of $f=300 \mathrm{~mm}$. The intensity patterns of the field transmitted from the $\mathrm{CS}_{2}$ cell are imaged on a detector (Beamview, Coherent Inc.) using an achromatic lens with a focal length of $f=60 \mathrm{~mm}$. The experimental results of the AV-LP-VFs (not shown here) reveal that the number
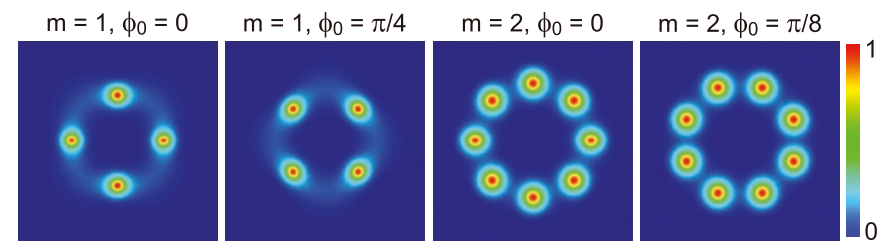

Figure $2 \mid$ Simulated collapsing patterns of AV-HP-VFs with different $m$ and $\phi_{0}$ at a propagation distance of $\zeta=0.36$ with random noise. 
and positions of the induced collapsing filaments have the uncertainty among the experiments, depending on the spatial random fluctuation of the input field. Any one of the measured collapsing patterns of the $m=1$ charge AV-HP-VFs, with four different $\phi_{0}$ $(=0, \pi / 8, \pi / 4,3 \pi / 8)$, consists of four deterministic filaments and exhibits a fourfold rotation $\left(C_{4}\right)$ symmetry (Fig. 3). The four patterns have no difference in nature, whereas their unique difference is that the patterns from Fig. $3 \mathrm{a}-\mathrm{d}$ are rotated counterclockwisely in sequence by an angle of $\pi / 8$. Figure 4 shows the measured collapsing patterns of AV-HP-VFs (with $\phi_{0}=0$ ) for $m=1,2,3,4$. The $m$ charge AV-HP-VF produces $4 m$ deterministic filaments, where $m=$ $1,2,3,4$, with the number of filaments being $4,8,12,16$, respectively. The collapsing pattern exhibits a $4 m$-fold rotation $\left(C_{4 m}\right)$ symmetry. In particular, the positions of filaments for any AV-HP-VF always coincide with those of the local linear polarizations in the azimuthal dimension. The collapsing patterns of the AV-HP-VF with a given $m$ and $\phi_{0}$ persist among experiments, implying that the collapsing patterns are indeed insensitive to the ransom noise. Moreover, the collapsing pattern of the AV-HP-VF is controllable and designable by engineering the spatial hybrid SoP structure.

\section{Discussion}

To understand the physics behind the collapse of AV-HP-VF, a cross-coupling model is presented (Fig. 5). Multiple filamentation requires the simultaneous spatial confinements in both the radial and azimuthal dimensions. The self-confinement in the radial dimension is due to the focal ring. However, it is of great interest and of crucial importance to reveal the mechanism behind the axial symmetry breaking that leads to the multiple filamentation. Therefore, we ignore the radial terms $\partial^{2} / \partial \rho^{2}$ and $\partial / \partial \rho$ in the transverse Laplacian $\nabla_{\perp}^{2}$, while concentrate only on the azimuthal term $\partial^{2} / \partial \phi^{2}$ in Eq. (3). The Kerr medium is divided into $N$ equal-length thin segments (each thin segment has a normalized length of $\Delta \zeta=L /$ $N)$, so that the nondepletion approximation is valid within any thin segment. After the focused AV-LP-VF described in Eq. (2) passing though the 1st segment, based on Eq. (3), $\psi_{H}$ and $\psi_{V}$ can be expressed as follows

$$
\begin{aligned}
& \left.\psi_{H}\right|_{\zeta=\Delta \zeta}=[A+j(B+C)] \cos \delta, \\
& \left.\psi_{V}\right|_{\zeta=\Delta \zeta}=[A+j(B+C)] \sin \delta,
\end{aligned}
$$

thereby obtaining

$$
\left.\left(\left|\psi_{H}\right|^{2}+\left|\psi_{V}\right|^{2}\right)\right|_{\zeta=\Delta \zeta}=A^{2}+(B+C)^{2},
$$

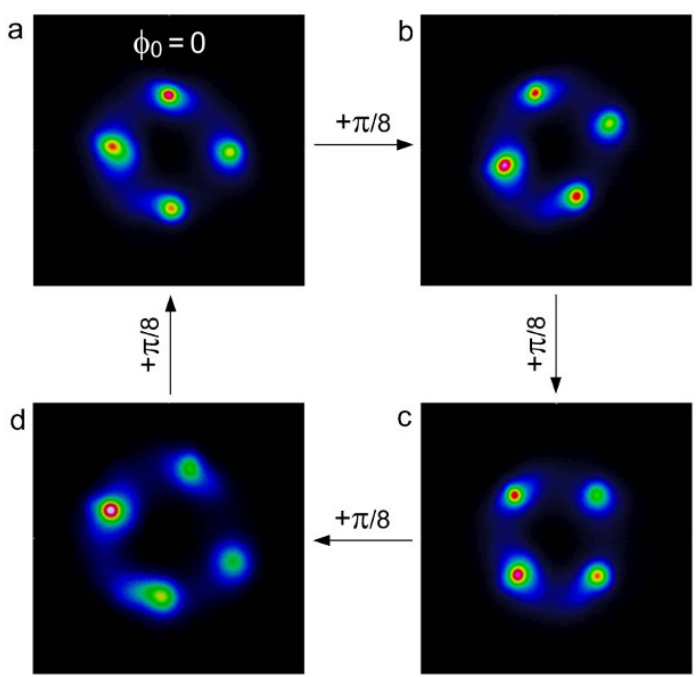

Figure $3 \mid$ Measured collapsing patterns of AV-HP-VFs with $\boldsymbol{m}=\mathbf{1}$ for different $\phi_{0}$. (a) $\phi_{0}=0$. (b) $\phi_{0}=\pi / 8$. (c) $\phi_{0}=\pi / 4$. (d) $\phi_{0}=3 \pi / 8$.
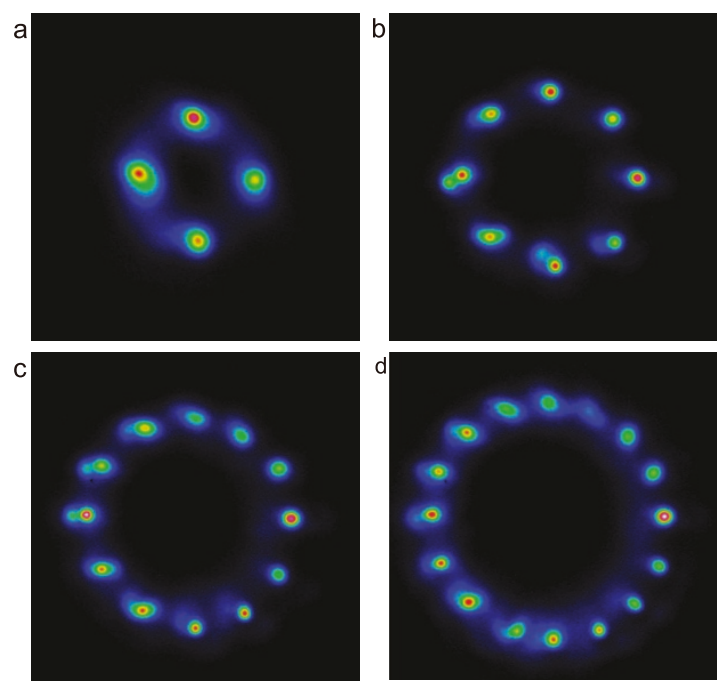

Figure $4 \mid$ Measured collapsing patterns of AV-HP-VFs with $\phi_{0}=0$ for different $m$. (a) $m=1$. (b) $m=2$. (c) $m=3$. (d) $m=4$.

where $B=-A m^{2} \Delta \zeta / 4$ and $C=\alpha P A^{3} \Delta \zeta / P_{C}$. Based on Eqs. (4) and (5), we can find: (i) the horizontal and vertical components are always held in phase, implying that spatial SoP distribution has no change; and (ii) the intensity distribution still exhibits axial symmetry. Therefore, a purely ideal AV-LP-VF can never produce the deterministic multiple filamentation, which is in excellent agreement with the simulation (the third row of Fig. 1). As such, any azimuthal position is equivalent or indistinguishable. In the presence of azimuthal perturbations, axial symmetry breaking will lead to the filamentation $^{1-5}$ (also see the fourth row of Fig. 1).

After the AV-HP-VF described in Eq. (1) passing through the 1st segment, based on Eq. (3), $\psi_{H}$ and $\psi_{V}$ can be expressed as follows

$$
\begin{aligned}
& \left.\psi_{H}\right|_{\zeta=\Delta \zeta}=\left\{A+j\left[B+(C / 3)\left(1+2 \cos ^{2} \delta\right)\right]\right\} \cos \delta, \\
& \left.\psi_{V}\right|_{\zeta=\Delta \zeta}=j\left\{A+j\left[B+(C / 3)\left(1+2 \sin ^{2} \delta\right)\right]\right\} \sin \delta,
\end{aligned}
$$

thereby obtaining

$$
\left.\left(\left|\psi_{H}\right|^{2}+\left|\psi_{V}\right|^{2}\right)\right|_{\zeta=\Delta \zeta}=A^{\prime}+B^{\prime} \cos (4 \delta),
$$

where

$$
\begin{gathered}
A^{\prime}=A^{2}+(B-C)^{2}-(B+5 C / 6)(C / 3), \\
B^{\prime}=-(B-5 C / 6)(C / 3) .
\end{gathered}
$$
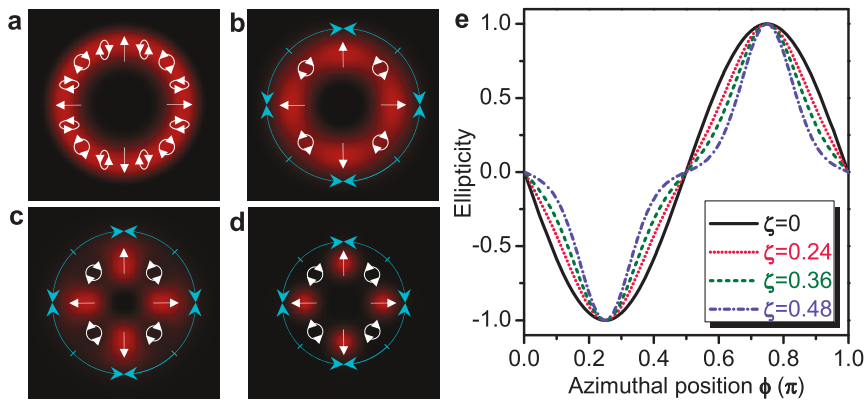

Figure 5 | Cross-coupling model for the collapse of AV-HP-VF. (a) The intensity and SoP distributions in the input plane. (b) Those behind the first segment. (c) Those behind the $n$th segment. (d) Those in the output plane. Arc arrows indicates the energy exchange directions. (e) The simulated evolution of $S_{3}$ of azimuthal hybrid SoPs for the AV-HP-VF with $m=1$ and $\phi_{0}=0$ (only the azimuthal range from $\phi=0$ to $\pi$ is shown). 

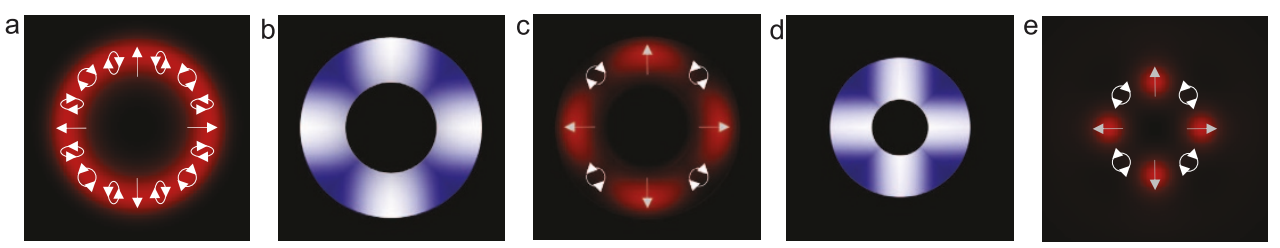

Figure 6 Azimuthal self-focusing model for the collapse of AV-HP-VF. (a) Input focused AV-HP-VF. (b) Induced azimuthal self-focusing lenses. (c) Axial-symmetry broken AV-HP-VF. (d) Induced azimuthal self-focusing lenses with the shorter "focal length". (e) Collapsing filaments.

Equation 7 suggests that the nonlinear cross-coupling results in the energy redistribution of AV-HP-VF in the azimuthal dimension (or the axial symmetry breaking). It should be pointed out that the intensity distribution still holds the axial symmetry in the absence of nonlinearity $\left(n_{2}=0 \rightarrow C=0 \rightarrow B^{\prime}=0\right)$. As an example, the AV-HPVF with $m=1$ and $\phi_{0}=0$ is considered (Figs. 5a-d). In the input plane $(\zeta=0)$, the focused AV-HP-VF has the azimuthally-invariant intensity distribution, while the azimuthally-variant hybrid SoP distribution (Fig. 5a). After the 1st segment, the cross-coupling results in the energy exchange in the azimuthal dimension toward the locations at $\phi^{\text {lin }}(n)$ from those at $\phi^{\text {cir }}(n)$ (Fig. 5b). As the propagation distance increases, more energy is transferred toward the locations at $\phi^{\text {lin }}(n)$ after the $j$ th segment (Fig. 5c). In the output plane (Fig. 5d), the cross-coupling leads to the energy almost converging into the azimuthal locations at $\phi^{\text {lin }}(n)$. The axial symmetry of the focused AVHP-VF is broken ultimately to form four deterministic filaments. From Eq. 6, the cross-coupling acquires an additional phase, which makes the phase difference between the horizontal and vertical components to not always preserve $\pi / 2$, resulting in the SoP evolution in contrast to AV-LP-VF. However, at two series of azimuthal locations, $\phi^{\text {lin }}(n)$ and $\phi^{\text {cir }}(n)$, the SoPs have no change. The simulated evolution of the azimuthal-variant hybrid SoPs, by the Stokes parameter $S_{3}$ characterizing the ellipticity ${ }^{30}$, during the nonlinear propagation of the AV-HP-VF with $m=1$ and $\phi_{0}=0$ confirmed the above prediction (Fig. 5e). As propagation distance increases, the local ellipticity has a tendency to decrease (i.e, the local polarization ellipse will become flatter). Except for at the special azimuthal locations of $\phi^{\text {lin }}(n)$ and $\phi^{\text {cir }}(n)$, the SoPs have indeed no change, like the above discussion from the cross-coupling model.

To understand the collapsing filaments, the azimuthal self-focusing model is also very beneficial (Fig. 6). The self-focusing phenomenon originates from the spatially variant refractive index change. In an isotropic Kerr medium under the same intensity, the induced refractive index change depends on the SoP of optical field $\left(\Delta n^{\text {lin }}\right.$ $>\Delta n^{\text {ell }}>\Delta n^{\text {cir }}$ ), where $\Delta n^{\text {lin }}, \Delta n^{\text {ell }}$, and $\Delta n^{\text {cir }}$ are the refractive index changes induced by the linearly, elliptically, and circularly polarized light, respectively ${ }^{35}$. As a result, the refractive index change induced by the AV-HP-VF is azimuthally variant, leading to the axial-symmetry breaking. The local linear and circular polarizations are located at $\phi^{\text {lin }}(n)$ and $\phi^{\text {cir }}(n)$ (where $\left.n=0,1,2,3\right)$ for the AV-HPVF with $m=1$ and $\phi_{0}=0$ (Fig. 6a). Therefore, the induced four azimuthal self-focusing lenses have their centers located at $\phi^{\text {lin }}(n)$ and their edges located at $\phi^{\text {cir }}(n)$ (Fig. 6b). The input field undergoes the azimuthal multiple-nucleation self-focusing, making the energy converge toward the four locations at $\phi^{\text {lin }}(n)$ (Fig. 6c). As the propagation distance increases, the "focal lengths" of the induced azimuthal selffocusing lenses become shorter (Fig. $6 \mathrm{~d}$ ), and then the energy converges ultimately into the four deterministic filaments (Fig. 6e). In particular, $\Delta n^{l i n}$ is greater than $50 \% \Delta n^{\text {cir }}$, which is the reason why $\mathrm{AV}-\mathrm{HP}-\mathrm{VF}$ can suppress the random noise (the robust feature) during the deterministic collapsing filamentation.

We have presented the theoretical prediction and conducted the experimental evidence on the novel collapsing behaviors of AV-HPVF in the self-focusing Kerr medium. The azimuthally-variant hybrid SoP structure is crucial in the axial symmetry breaking. The collapsing filamentation has the controllable and robust features.
The $m$-charge AV-HP-VF produces $4 m$ filaments with $4 m$-fold rotation $\left(C_{4 m}\right)$ symmetry. The number of multiple filaments is a function solely of $m$, and their locations are determined by both $m$ and $\phi_{0}$. Our results suggest that engineering the hybrid SoP structure can tame the randomicity and then realize the designable collapsing filamentation. This study only concentrated on the collapsing behavior; thus, the higher-order nonlinearity, which is important in the postcollapse process, has been neglected. Of course, we also simulated the multiple filamentation when the higher-order Kerr effect is considered, the results indicate a novel feature that the relative phase difference among the multiple filaments is stable and insensitivity to the input fluctuations of the pulse energy, which is quite different from the reported fact that the relative phase difference is instable and sensitivity to the small fluctuations of the pulse energy, i.e., loss of phase of collapse $\mathrm{e}^{36}$. Our idea may offer an alternative route to produce the controllable and robust multiple filamentation in other nonlinear systems, thereby facilitating the development of additional surprising applications. This work has the great significance that it not only opens a new window for manipulating the optical field and facilitates to push the related researches, but also has a certain reference value for manipulating the different kinds of field.

\section{Methods}

Generation of femtosecond vector fields. We follow the same method as Ref. 30 for creating the vector fields required in this study. The used light source is a Ti:sapphire regenerative amplifier femtosecond laser system (Coherent Inc.), which provides a fundamental Gaussian mode with a central wavelength of $800 \mathrm{~nm}$, a pulse duration of $35 \mathrm{fs}$, and a repetition rate of $1 \mathrm{kHz}$. An achromatic $1 / 2$ wave plate and a broadband polarized beam splitter were used to control incident laser fluence on the sample surface. Another achromatic $1 / 2$ wave plate was used to change the polarization direction of the incident femtosecond laser into the vector field generation unit, where all the elements are achromatic to suppress pulse broadening as much as possible. The created AV-HP-VFs and AV-HP-VFs have a "top-hat-like" spatial profile, excluding a central singularity caused by the SoP uncertainty, with a radius of $1.5 \mathrm{~mm}$, a pulse energy of $6.0 \mu \mathrm{J}$, and a pulse duration of $\sim 65 \mathrm{fs}$.

1. Robinson, P. A. Nonlinear wave collapse and strong turbulence. Rev. Mod. Phys. 69, 507-573 (1997)

2. Bergé, L. Wave collapse in physics: principles and applications to light and plasma waves. Phys. Reports 303, 259-370 (1998).

3. Fibich, G. \& Ilan, B. Vectorial and random effects in self-focusing and in multiple filamentation. Physica D 157, 112-146 (2001).

4. Couairon, A. \& Mysyrowicz, A. Femtosecond filamentation in transparent media. Phys. Reports 441, 47-189 (2007).

5. Bergé, L., Skupin, S., Nuter, R., Kasparian, J. \& Wolf, J. P. Ultrashort filaments of light in weakly ionized, optically transparent media. Rep. Prog. Phys. 70, 1633-1713 (2007).

6. Chiao, R. Y., Garmire, E. \& Townes, C. H. Self-trapping of optical beams. Phys. Rev. Lett. 13, 479-482 (1964).

7. Bespalov, V. I. \& Talanov, V. I. Filamentary structure of light beams in nonlinear liquids. JETP Lett. 3, 307-312 (1966).

8. Kelley, P. L. Self-focusing of optical beams. Phys. Rev. Lett. 15, 1005-1008 (1965)

9. Varma, S., Chen, Y. H. \& Milchberg, H. M. Trapping and destruction of longrange high-intensity optical filaments by molecular quantum wakes in air. Phys. Rev. Lett. 101, 205001 (2008).

10. Braun, A., Korn, G., Liu, X., Du, D., Squier, J. \& Mourou, G. Self-channeling of high-peak-power femtosecond laser pulses in air. Opt. Lett. 20, 73-75 (1995).

11. Kasparian, J. \& Wolf, J. P. Physics and applications of atmospheric nonlinear optics and filamentation. Opt. Express 16, 466-493 (2008).

12. Kasparian, J., Rodriguez, M., Méjean, G., Yu, J., Salmon, E., Wille, H., Bourayou, R., Frey, S., André, Y. B., Mysyrowicz, A., Sauerbrey, R., Wolf, J. P. \& Wöste, L. White-light filaments for atmospheric. Science 301, 61-64 (2003). 
13. Kolesik, M., Wright, E. M. \& Moloney, J. V. Femtosecond filamentation in air and higher-order nonlinearities. Opt. Lett. 35, 2550-2552 (2010).

14. D’Amico, C., Houard, A., Franco, M., Prade, B., Mysyrowicz, A., Couairon, A. \& Tikhonchuk, V. T. Conical forward THz emission from femtosecond-laser-beam filamentation in air. Phys. Rev. Lett. 98, 235002 (2007).

15. Alfano, R. R. \& Shapiro, S. L. Emission in the region 4000 to $7000 \AA$ via fourphoton coupling in glass. Phys. Rev. Lett. 24, 584-587 (1970).

16. Hauri, C. P., Kornelis, W., Helbing, F. W., Heinrich, A., Couairon, A., Mysyrowicz, A., Biegert, J. \& Keller, U. Generation of intense, carrier-envelope phase-locked few-cycle laser pulses through filamentation. Appl. Phys. B 79, 673-677 (2004).

17. Rohwetter, P., Kasparian, J., Stelmaszczyk, K., Hao, Z., Henin, S., Lascoux, N., Nakaema, W. M., Petit, Y., Queißer, M., Salamé, R., Salmon, E., Wöste, L. \& Wolf, J. P. Laser-induced water condensation in air. Nature Photon 4, 451-456 (2010).

18. Belgiorno, F., Cacciatori, S. L., Clerici, M., Gorini, V., Ortenzi, G., Rizzi, L., Rubino, E., Sala, V. G. \& Faccio, D. Hawking radiation from ultrashort laser pulse filaments. Phys. Rev. Lett. 105, 203901 (2010).

19. Jin, Z., Zhang, J., Xu, M. H., Lu, X., Li, Y. T., Wang, Z. H., Wei, Z. Y., Yuan, X. H. \& $\mathrm{Yu}, \mathrm{W}$. Control of filamentation induced by femtosecond laser pulses propagating in air. Opt. Express 13, 10424-10430 (2005).

20. Dubietis, A., Tamošauskas, G., Fibich, G. \& Ilan, B. Multiple filamentation induced by input-beam ellipticity. Opt. Lett. 29, 1126-1128 (2004).

21. Grow, T. D. \& Gaeta, A. L. Dependence of multiple filamentation on beam ellipticity. Opt. Express 13, 4594-4599 (2005).

22. Pfeifer, T., Gallmann, L., Abel, M. J., Neumark, D. M. \& Leone, S. R. Circular phase mask for control and stabilization of single optical filaments. Opt. Lett. 31, 2326-2328 (2006).

23. Kandidov, V. P., Aközbek, N., Scalora, M., Kosareva, O. G., Nyakk, A. V., Luo, Q., Hosseini, S. A. \& Chin, S. L. A method for spatial regularisation of a bunch of filaments in a femtosecond laser pulse. Quantum Electronics 34, 879-880 (2004).

24. Hao, Z. Q., Stelmaszczyk, K., Rohwetter, P., Nakaema, W. M. \& Woeste, L. Femtosecond laser filament-fringes in fused silica. Opt. Express 19, 7799-7806 (2011).

25. Hao, Z. Q., Zhang, J., Xi, T. T., Yuan, X. H., Zheng, Z. Y., Lu, X., Yu, M. Y., Li, Y. T., Wang, Z. H., Zhao, W. \& Wei, Z. Y. Optimization of multiple filamentation of femtosecond laser pulses in air using a pinhole. Opt. Express 15, 16102-16109 (2007).

26. Kandidov, V. P., Akozbek, N., Scalora, M., Kosareva, O. G., Nyakk, A. V., Luo, Q., Hosseini, S. A. \& Chin, S. L. Towards a control of multiple filamentation by spatial regularization of a high-power femtosecond laser pulse. Appl. Phys. B 80, 267-275 (2005)

27. Trisorio, A. \& Hauri, C. P. Control and characterization of multiple circularly polarized femtosecond filaments in argon. Opt. Lett. 32, 1650-1652 (2007).

28. Fibich, G. \& Ilan, B. Deterministic vectorial effects lead to multiple filamentation. Opt. Lett. 26, 840-842 (2001)
29. Zhan, Q. Cylindrical vector beams: from mathematical concepts to applications. Adv. Opt. Photon. 1, 1-57 (2009).

30. Wang, X. L., Li, Y. N., Chen, J., Guo, C. S., Ding, J. P. \& Wang, H. T. A new type of vector fields with hybrid states of polarization. Opt. Express 18, 10786-10795 (2010).

31. Vuong, L. T., Grow, T. D., Ishaaya, A. A., Gaeta, A. L., 't Hooft, G. W., Eliel, E. R. \& Fibich, G. Collapse of optical vortices. Phys. Rev. Lett. 96, 133901 (2006).

32. Wang, X. L., Chen, J., Li, Y. N., Ding, J. P., Guo, C. S. \& Wang, H. T. Optical orbital angular momentum from the curl of polarization. Phys. Rev. Lett. 105, 253602 (2010).

33. Fibich, G. \& Gaeta, A. L. Critical power for self-focusing in bulk media and in hollow waveguides. Opt. Lett. 25, 335-337 (2000).

34. Ishaaya, A. A., Vuong, L. T., Grow, T. D. \& Gaeta, A. L. Self-focusing dynamics of polarization vortices in Kerr media. Opt. Lett. 33, 13-15 (2008).

35. Sutherland, R. L. Handbook of Nonlinear Optics (second Edition, Marcel Dekker, Inc., 2003).

36. Shim, B., Schrauth, S. E., Gaeta, A. L., Klein, M. \& Fibich, G. Loss of phase of collapsing beams. Phys. Rev. Lett. 108, 043902 (2012).

\section{Acknowledgements}

This work was supported by the 973 Program of China under Grant No. 2012CB921900, and the National Natural Science Foundation of China under Grants 10934003.

\section{Author contributions}

S.M. Li presented idea, performed the experiments, carried out simulations, and wrote the manuscript. Y.N. Li assisted with the experiments and the discussion of mechanism. X.L. Wang presented the method for generating the vector fields. L.J. Kong and K. Lou assisted with the experiments and the discussion. C.H. Tu provided technical support. Y.J. Tian assisted with the discussion of mechanism and wrote the manuscript. H.T. Wang planned the project, presented the idea and the mechanism, guided and supervised the simulations and experiments, and wrote the manuscript.

\section{Additional information}

Competing financial interests: The authors declare no competing financial interests.

License: This work is licensed under a Creative Commons

Attribution-NonCommercial-ShareAlike 3.0 Unported License. To view a copy of this license, visit http://creativecommons.org/licenses/by-nc-sa/3.0/

How to cite this article: Li, S.M. et al. Taming the Collapse of Optical Fields. Sci. Rep. 2 , 1007; DOI:10.1038/srep01007 (2012). 